\title{
Medicina tradicional y fitoterapia una alternativa para el mejoramiento de la salud en Guatemala
}

\author{
Sully M. Cruz* \\ Facultad de Ciencias Químicas y Farmacia, Universidad de San Carlos de Guatemala, Guatemala. \\ *Autor al que se dirige la correspondencia: sullycv@hotmail.com \\ Recibido: 12 de agosto 2015 / Revisión: 13 de agosto 2015 / Aceptado: 13 de mayo 2016 / Disponible en línea: 17 de junio 2016
}

\section{Resumen}

$\mathrm{E}$ 1 empleo de las plantas medicinales con fines curativos es una práctica que se ha utilizado ancestralmente. Durante mucho tiempo los remedios naturales fueron el principal recurso disponible, esto hizo que se profundizara en el conocimiento de las especies vegetales, surgiendo así como una disciplina la fitoterapia. En todo el mundo, la medicina tradicional, complementaria o no convencional ha constituido un pilar importante en la prestación de servicios de salud. Muchos países reconocen actualmente la necesidad de elaborar un enfoque coherente e integral de la atención de salud que facilite el acceso de la medicina tradicional de manera segura, respetuosa, asequible y efectiva, por lo que surge la estrategia de la Organización Mundial de Salud 2002-2005, que por su importancia fue actualizada para 2014-2023. En Guatemala existe una práctica tradicional de uso de plantas medicinales, además se cuenta con un Programa Nacional de Medicina Tradicional y con el Vademécum Nacional de Plantas Medicinales, el cual contiene la información sobre plantas medicinales validadas y con suficiente evidencia científica. Derivado del análisis de la situación del sistema de salud, se plantea como una de las estrategias para mejorar el acceso, la calidad de la atención y la promoción de la salud, un abordaje intercultural, dándole un nuevo enfoque al sistema de salud de una forma integrada, incluyente y pluricultural, que promueva la calidad en el servicio y que responda a las necesidades de la comunidad con los recursos que le brinda su ambiente.

\begin{abstract}
$\mathrm{T}$ raditional use of herbal medicines refers to the long historical use of these medicines, for a long time natural remedies were the main resource available, making the knowledge of plant species an emerging discipline, phytotherapy. Worldwide, traditional, complementary or unconventional medicine has constituted an important pillar in the provision of health services, many countries recognize the need to develop a coherent and comprehensive approach in health care, facilitating access of traditional medicine in a safe, friendly, affordable, and effective way. From this reason arise the strategy of the World Health Organization 2002-2005, because of its importance it was updated for 2014-2023. In Guatemala there is a traditional practical use of medicinal plants, also there is a National Program on Traditional Medicine and the National Vade mecum on Medicinal Plants, which contain validated information about medicinal plants and with sufficient scientific evidence. Derived from the analysis of the national health system, medicinal plants are proposed as one of the strategies to improve access to health care quality, and health promotion of intercultural approach, giving a new proposal to health care systems as integrated, inclusive, accessible and multicultural that promotes quality in service and to answer the needs of the community with the resources provided by the environment.
\end{abstract}




\section{Introducción}

En muchas partes del mundo, las instancias normativas, los profesionales de la salud y el público están enfrentando asuntos relativos a la seguridad, eficacia, calidad, disponibilidad, preservación y reglamentación tanto de la medicina tradicional y complementaria (MTC), como la utilización racional de los productos naturales medicinales comercializados. La utilización de la MTC sigue siendo amplia en la mayoría de los países, y está creciendo rápidamente. Al mismo tiempo, el interés por la MTC se está extendiendo más allá de los productos naturales, y abarca también las prácticas y los profesionales.

El público y los usuarios de servicios de salud en todo el mundo siguen incluyendo la MTC y los productos naturales entre sus decisiones relacionadas con la salud. El aumento del interés por la MTC exige su integración en los sistemas de salud. Las instancias normativas y los usuarios deberían examinar de qué manera la MTC puede mejorar la experiencia del paciente y la salud de la población. Se deben considerar importantes cuestiones relativas al acceso, así como a la población y la salud pública.

Cuando se analizan las causas de la mortalidad general en Guatemala, para el período 2001-2012, se observa que el perfil epidemiológico sufre una transición de las causas infecciosas a las causas crónicas (no transmisibles) y las lesiones externas (muchas de estas son producto de la violencia).

Para el abordaje de los principales problemas de salud en Guatemala se propone por parte del Ministerio de Salud Pública y Asistencia Social (MSPAS) la implementación de la estrategia de atención primaria en salud renovada, la cual integra la organización del sistema de atención a la salud y el desarrollo social, para actuar sobre los determinantes de la salud bajo principios de equidad, justicia social, respeto a la diversidad cultural, cobertura y acceso universal, promoción de la salud, prevención de enfermedades, atención oportuna y resolutiva, empleo de tecnologías apropiadas e impulso a la participación social y comunitaria.

Por lo anteriormente indicado se sugiere que la implementación de la MTC y la utilización de los productos naturales constituyen una opción viable para la población ya que hay aceptación, interés y disponibilidad y ha formado parte de la cultura desde tiempos ancestrales.

\section{Contenido}

La medicina es parte de la cultura de un pueblo. No hay pueblo, que no haya desarrollado algún sistema de medicina, es decir, un cuerpo ideológico o doctrinario acerca de la vida y la muerte, la salud y la enfermedad, y más concretamente sobre las causas de las afecciones, la manera de reconocerlas y diagnosticarlas, así como las formas o procedimientos para aliviar, curar o prevenir las enfermedades, y además para preservar y promover la salud (Cotton, 2004; Organización Mundial de la Salud [OMS], 1991). Para la OMS, la salud es un estado de completo bienestar físico, mental y social, y no solamente la ausencia de afecciones o enfermedades. Es el bienestar biológico, psicológico, social y espiritual del individuo y de la comunidad. También es la expresión individual y colectiva de calidad de vida y bienestar; y producto de las determinaciones sociales. Comprende el conjunto de condiciones biológicas, psicológicas, materiales, sociales y culturales; que tienen como determinantes, entre otros, la alimentación, la vivienda, el saneamiento básico, el medio ambiente, el trabajo, la renta, la educación, el transporte y el acceso a bienes y servicios esenciales. Su realización define la condición de estar y permanecer sano, ejerciendo cada cual a plenitud sus capacidades potenciales a lo largo de cada etapa de la vida. Se alcanza a través del esfuerzo colectivo, intersectorial y participativo de todos y todas, orientado hacia la conquista y defensa de oportunidades equitativas materiales y sociales de vida, para mejorar el bienestar social y económico de toda la población (OMS, 1991, 2002).

La salud, es además, un derecho humano que asiste a todas las personas, indistintamente de su género, religión, color, etnia o idioma. Es el derecho que existe a la oportunidad de recibir atención de salud en lo personal, lo familiar y en la comunidad, y es un deber y responsabilidad del Estado, asegurar efectivamente los derechos a la salud de las poblaciones indígenas (OMS, 2002).

Actualmente, según la OMS alrededor de cuatro mil millones de personas, aproximadamente el 80\% de la población mundial, utiliza las plantas como remedio, siendo varias las razones por las que socialmente el uso de las plantas medicinales está tomando un cariz hasta hace unas décadas insospechado, entre ellas como respuesta a una medicina alopática, muchas veces iatrogénica, en otros casos por el reducido o inexistente acceso económico de la población a los medicamentos de síntesis, aunado al hecho irrefutable de que los países 
en vías de desarrollo, no tienen una industria farmacéutica poderosa.

La utilización de plantas medicinales como materia prima para la producción de extractos o para el aislamiento de sustancias naturales puras, representa un sector en franca expansión. Para 1995 según datos estadísticos, Según datos estadísticos de 1995, el mercado de plantas medicinales y preparados fitofarmacéuticos alcanzaba la cifra de 6,500 millones de USD en Europa, 4,600 millones de USD en Asia y 1,500 millones de USD en América del Norte, totalizando 12,600 millones de USD. En Alemania, el porcentaje de población que emplea medicamentos fitoterápicos ha experimentado un aumento, entre 1970 y 1997, de entre un 4\% y un 92\% según las patologías (Cañigueral, 2002; Cañigueral \& Vila, 2003).

Esta creciente utilización, propiciada por el regreso general de la sociedad a lo natural, va más allá de una simple moda y no se debe solamente a un aumento en el interés por los "tratamientos naturales" de los problemas de salud, sino también a la creciente evidencia sobre su seguridad y eficacia. Entre las razones que justifican el creciente interés por las plantas medicinales y por los preparados fitoterápicos en los países desarrollados están el cambio en las preferencias del consumidor, que muestra su predilección por los productos naturales, la escasez de nuevos descubrimientos, mediante los procesos tradicionales de síntesis química, de moléculas farmacológicamente activas con posible aplicación terapéutica y los efectos secundarios derivados del uso correcto de algunos fármacos sintéticos (talidomida, clioquinol, hexaclorofeno, etc) (Bonati, 1990; Cañigueral, 2002; Cañigueral \& Vila, 2003).

En los países en vías de desarrollo, donde vive el $75 \%$ de la población mundial, se consumen menos del $15 \%$ del mercado total de medicamentos. Las plantas medicinales representan, por tanto, el único recurso terapéutico disponible para los sectores más desfavorecidos de esta población. Teniendo en cuenta el crecimiento constante de la población en los países del tercer mundo, la importancia de las plantas medicinales y de su uso, con diferentes niveles de industrialización, es cada vez mayor en los países en vías de desarrollo (Cañigueral \& Vila, 1998; Mendoza, León \& Figueroa-Hernández, 2005).

Dentro de este virtual auge de las llamadas terapias naturales, estamos recién en los pasos iniciales para una correcta integración, avance y difusión de éstas al servicio de la salud humana, frente a un futuro en el cual no es ilusorio imaginar que paulatinamente los países de cualquier continente aplicarán no sólo el marco legal-normativo para plantas medicinales en su política de salud, sino también serán de rigor las enseñanzas universitarias oficiales de disciplinas que ahora en el presente (exceptuando algunos países) son marginales, tales como: fitoterapia, acupuntura, homeopatía, terapias florales, fitoterapia, hidroterapia, terapia ortomolecular, entre otras del actual grupo denominado medicinas alternativas (Cañigueral, 2002; Tyler, 1999).

El avance de la fitoterapia como parte esencial del sistema de salud se logrará con la participación multidisciplinaria de botánicos, químicos, farmacólogos, médicos, industriales, agrónomos, forestales, técnicos, ecologistas, educadores, etcétera, además de una acción multisectorial, de tal modo que se constituya en determinado momento no sólo en una terapia complementaria del médico convencional autodidacta en esta disciplina sino fundamentalmente en una alternativa de salud al servicio de los pueblos, en un marco de ética y filosofía humanistas (Cañigueral \& Vila, 2003; Obregón, 2000).

Si se efectuara un estudio comparativo basado en las prácticas medicinales de la cultura indígena tradicional de la actualidad, este revelaría sin duda alguna que al $100 \%$ de los indígenas utilizan las plantas medicinales en sus tratamientos curativos, pero al mismo tiempo las entrelazan estrechamente con muchas creencias supersticiosas y religiosas. Por otra parte, el estudio de la cultura ladina mestiza constituye un cuadro diferente y más complicado, debido a los diversos grados de ladinización, que fueron surgiendo como fruto de un complejo proceso de conquista y colonización de Guatemala, constituyéndose así los ladinos en una población heterogénea, en sus manifestaciones físicas y culturales, con una estructura social y étnica desigual y diferenciada presentando contradicciones de origen económico, tratando de mantener un estatus étnico y social que se originó entre los mestizos rurales, urbanos y criollos, los cuales formaron un solo bloque frente al indígena. Por lo que resulta muy difícil y casi imposible puntualizar el grado exacto de los cambios culturales en un individuo, mucho menos en un grupo de individuos. De los estudios que se han hecho, así como de los resultados obtenidos a través de entrevistas y diversas fuentes, se ha establecido una relación entre el tipo de tratamiento medicinal utilizado por un individuo y el grado de ladinización, la situación económica y el grado de educación del individuo (Bruhn \& Holmstedt, 1981; Eder \& García, 2003; Villatoro, 1982). 
Guatemala es un país de contrastes y de diversidad cultural, determinada esta última por la existencia de una población pluricultural, pluriétnica y multilingüe, lo que se manifiesta a través de la coexistencia de cuatro grupos culturales principales: Los de origen maya, los garífunas, los xincas y los mestizos. Para el año 2012 la población proyectada fue $15,073,397$ habitantes, con una densidad poblacional de $138 \mathrm{hab} / \mathrm{km}^{2}$; el $51.5 \%$ de la población vive en áreas rurales y el $23 \%$ en el área metropolitana, lo que implica una mayor demanda de servicios de salud en estas áreas. El país presenta los siguientes índices: Analfabetismo 18\%, pobreza 56\% y pobreza extrema $24 \%$. En el área metropolitana existe una intensa y desordenada urbanización, la cual contribuye a que la población sea más vulnerable a riesgos, enfermedades transmisibles infecciosas y desarrollo de enfermedades crónicas degenerativas. En el área rural una población más dispersa dificulta la prestación de los servicios de salud (Instituto Nacional de Estadística [INE], 2009; MSPAS, 2012; Secretaría de Planificación y Programación de la Presidencia [SEGEPLAN], 2011).

El sistema de salud de Guatemala está segmentado en varios sub-sistemas, los cuales brindan atención a diferentes usuarios. A pesar del funcionamiento de los sub-sistemas no existe la cobertura total. Asimismo, los subsistemas en su interior están fragmentados, con vacíos reales de integración y coordinación lo cual posibilita la dualidad de funciones, mayor consumo de recursos, bajo rendimiento y grados altos de ineficiencia e ineficacia. Aunado a esto, se observa también una débil función rectora del Ministerio de Salud Pública y Asistencia Social (MSPAS, 2012).

Se podría caracterizar que actualmente el sector salud es de naturaleza mixta, conformado por el MSPAS y el Instituto Guatemalteco de Seguridad Social (IGSS), el Sector Privado, la Sanidad Militar y un significativo sector, principalmente en el interior del país, que practica la medicina tradicional basada en prácticas culturales ancestrales. En el análisis histórico se evidencia que el tema del financiamiento de la salud en Guatemala, no ha sido una preocupación institucional y responde al comportamiento de la oferta política expuesta en las diferentes coyunturas de Gobierno. El principal problema ha sido la asignación de recursos y manejo del gasto, que a lo largo de los años no se ha logrado las condiciones para fortalecer al Sistema de Salud Pública de Guatemala y satisfacer la demanda de la población (MSPAS, 2012).

Históricamente ha existido una tendencia a la segmentación de la población por su capacidad de pago o su inserción en el proceso productivo (los pobres e indigentes atendidos por el MSPAS, los asalariados por el IGSS, y la población de mayores recursos por el sector privado. No existe pues una organización del sistema alrededor de funciones y de hecho cada una de estas instituciones regula, financia y provee servicios (Cotton, 2004).

La cobertura de los servicios de salud para muchas poblaciones del área rural se encuentra extremadamente limitada. Estas poblaciones solamente reciben del Programa de Extensión de Cobertura (PEC) un paquete básico que no se ha modificado en más de 15 años. En algunos casos dicho paquete consiste en, una visita mensual única del médico o enfermera profesional a la comunidad. Dicho personal es contratado a través de las prestadoras y administradoras de los servicios de salud (organizaciones no gubernamentales, [ONG]) con funciones mayoritariamente curativas y poco preventivas. En el tema del recurso humano, son insuficientes y el equipo que utilizan está en obsolescencia en los tres niveles de atención. El MSPAS cuenta con pocos incentivos para reclutar y mantener el personal capacitado en especialidades (de salud pública y clínicas) que fortalezcan el enfoque de la salud integral en la práctica (MSPAS, 2012).

El actual presupuesto del MSPAS es totalmente insuficiente para brindar los servicios mínimos de salud a toda la población. El presupuesto asignado a esta institución para el año 2012 representa el 1.08\% del producto interno bruto (PIB). La red de servicios de salud del MSPAS se encuentra con problemas de organización, categorización, legalización, financiamiento, políticas de desarrollo del recurso humano, equipamiento e insumos médicos (MSPAS, 2012).

El Sistema de Salud de Guatemala tiene como propósito trabajar por la atención primaria de salud, dentro de la normativa de salud, se cuentan 21 programas de salud, cada uno de ellos con lineamientos de ejecución específicos para cada ciclo de vida, sin embargo, la mayoría de sus acciones se han centrado en el tratamiento de la enfermedad y son pocos los esfuerzos por establecer programas de prevención. Se puede decir que el sistema de salud se formó como una mezcla de modelos de salud occidentales, que han ido orientando sus acciones hacia determinados procesos o grupos de enfermedades, no se tiene un enfoque integral de abordar la salud (MSPAS, 2012).

Los indicadores de salud son alarmantes, Guatemala es una de las naciones latinoamericanas, que tiene los índices más altos de desnutrición crónica, a nivel 
mundial está dentro de los primeros cinco lugares, con un $49.3 \%$ de acuerdo con la Encuesta Nacional de Salud Materno-Infantil (ENSMI) 2002. Según estadísticas de la ENSMI 2008-2009 se indica que la desnutrición crónica en niños menores de 5 años, corresponde a $43.4 \%$, mientras que datos proporcionados por la OMS, se indica que la desnutrición es aún mayor llegando a un $49.8 \%$; también es importante resaltar las principales causas de mortalidad en menores de 5 años, entre las cuales sobresale la neumonía y la bronconeumonía (164 por cada 100,000 habitantes) y en las mujeres hay una alto riesgo de morir durante el embarazo, parto y puerperio (razón de mortalidad materna de 140 por cien mil nacidos vivos).

Las enfermedades de transmisión vectorial constituyen una problemática seria, durante el 2011, se reportaron 3,281 casos de dengue, de los cuales 29 casos fueron confirmados como dengue hemorrágico. Respecto a la malaria se concentra en dos departamentos, Escuintla y Zacapa y dentro de estos, en los municipios de La Gomera (64\%) y Gualán (24\%). Según reportaje presentado en Prensa Libre (Muñoz, 2016) se han confirmado un total de 90 casos de zika, y se reportan 276 casos sospechosos, según datos proporcionados por el MSPAS, siendo los departamentos de Suchitepéquez y Zacapa los que presentan mayor número de casos.

El 90\% del agua superficial (ríos, manantiales y lagos) presenta diversos grados de contaminación fecal, igualmente el agua entubada reporta contaminación ya que se ha detectado que la mayoría de municipalidades no realiza acciones para clorar el agua de consumo humano, lo que genera enfermedades transmitidas por agua y alimentos. Guatemala presenta enfermedades infecciosas principalmente atribuidas a la carencia de condiciones sanitarias y el desarrollo de enfermedades crónicas degenerativas y de estilos de vida, los que se suman a los riesgos ambientales (INE, 2009).

Garantizar la salud en Guatemala se ha convertido en un gran problema por falta de planes estratégicos y por el modelo adoptado de tipo biologista, curativo, reactivo y desintegrado, esto se evidencia a lo largo del tiempo ya que persisten los problemas de cobertura, accesibilidad y calidad en la atención y los servicios que se prestan, por lo que hay que considerar la participación multisectorial en la planeación, formulación y ejecución de las acciones para el mejoramiento del sistema de salud y la introducción de elementos de desarrollo comunitario y de salud pública.

El analfabetismo, la pobreza, el desempleo, la carencia de tierra y territorio, las altas tasas de mor- bilidad por causas evitables y las grandes limitantes obligan a despojarse de las pretensiones de objetividad y universalismo del sistema médico oficial y adoptar la fitoterapia como una opción viable para la población dentro del sistema de salud.

En tanto que la población dispone y utiliza distintos elementos terapéuticos provenientes de distintas tradiciones culturales, se requiere de una visión pluralista al interior del sistema. Las opciones terapéuticas de la gente son, por decirlo de alguna forma "democráticas" y que el sistema debe ser consecuente con esta realidad (Balick, 1990; Bruhn \& Holmstedt, 1981; OMS, 1991).

En Guatemala existe el Programa Nacional de Medicina Tradicional y Alternativa, el cual tiene como fin contribuir a la creación de condiciones políticas y estratégicas para el reconocimiento, valoración y rescate de la medicina popular tradicional y alternativa de atención; y del desarrollo del enfoque intercultural en salud, desde un abordaje en los niveles políticos, técnico normativo y operativo. Sin embargo, este programa ha sido ignorado y muy poco tomado en cuenta por los diferentes sectores, no ha logrado una incidencia eficaz dentro del sistema de salud para una integración y armonización que permita su incorporación formal en la solución de la problemática. Hay esfuerzos de lograr que tanto el sistema médico occidental como el etnomédico maya puedan convivir en mutuo respeto y entendimiento con acciones conjuntas, pero falta mucho trabajo por hacer, se necesita mayor participación, divulgación del programa y contar con el personal capacitado, ya que al estar integrado dicho programa por una sola persona, no ha logrado los recursos, impactos y resultados que le permitan avanzar como se esperaría.

Un avance importante fue la publicación del $\mathrm{Va}$ demécum Nacional de Plantas Medicinales obra realizada por Armando Cáceres (2009) de la Universidad de San Carlos y avalada por el MSPAS, el cual brinda información científica de especies medicinales, por lo que se constituye en una herramienta valiosa de referencia para que el personal de salud y la comunidad en general puedan utilizar apropiadamente las plantas medicinales en el tratamiento de enfermedades comunes basadas en la evidencia.

En general el sistema de medicina tradicional comunitaria ha sobrevivido con los servicios de los chamanes, los curanderos, los hierberos, los hueseros, los ancianos, las comadronas que desde la sabiduría de la cultura maya utilizan consejos, rituales, baños, plantas medicinales y productos animales y minerales para solucionar sus problemas de salud. 
Pero no se ha cuantificado estadísticamente, la mayoría de la población ha utilizado por muchos años incluso desde la época precolombina, las prácticas y medicinas tradicionales para la recuperación de su salud, lo cual todavía es una práctica cotidiana ante la falta de servicios y ante el abuso de los costos para acceder a las formas occidentales de curación. Así también, no existen registros estadísticos de la cobertura dada por las ONG, considerándose por parte del MSPAS, que estas tienen una cobertura aproximada del 18\% de la población, pero que con frecuencia atienden a grupos que también reciben atención de otras instituciones (Cotton, 2004).

Acorde a la Iniciativa de Salud de los Pueblos Indígenas (Sapia), la salud expresa relaciones dinámicas entre componentes inseparables, en lo individual (físico, mental, espiritual y emocional), lo colectivo (político, económico, cultural y social) y entre lo natural y lo social. La concepción de los pueblos indígenas respecto a la salud, está ligada a una perspectiva integral del mundo, como consecuencia, se prefiere definir la salud en términos de un bienestar integral que incorpora, infaltablemente, la dimensión espiritual. De esta manera, el concepto de salud integral abarca, el bienestar biológico, el psicológico, el social y el espiritual del individuo y de su comunidad en condiciones de equidad (Cotton, 2004).

Visto así, la salud de las poblaciones indígenas es una resultante de la integración de elementos fundamentales, como el acceso a la tierra, el nivel de participación en la sociedad nacional y la libertad para vivir su propia singularidad cultural en lo que respecta a su alimentación, vestimenta, vivienda, trabajo, educación $\mathrm{y}$, especialmente, sus posibilidades de acceso, acorde al derecho que les asiste, a los servicios públicos de atención de la salud en condiciones de equidad y dignidad (Bruhn \& Holmstedt, 1981; Gómez-Pompa, 1993).

Guatemala es un país que posee una gran riqueza natural de especies vegetales, lo cual ha dado lugar al uso de plantas en diferentes grupos étnicos de la población, por su accesibilidad y bajo costo. Desde épocas prehispánicas, a un sin número de plantas de la flora guatemalteca se les atribuyen propiedades medicinales, las cuales han sido adjudicadas en base a experiencias y uso tradicional (Cáceres, 1996; Martínez, 1964). Además por ser un país que presenta un rico acervo de conocimientos populares, obtenidos de una herencia cultural acumulada a través de su historia, se puede decir que cada grupo social o étnico ha seleccionado sus elementos, y los ha jerarquizado de acuerdo con sus necesidades, las cuales han sido condicionadas por el ambiente y su cultura.

Respecto a las creencias, prácticas y recursos médicos, se puede decir que cada grupo étnico ha seleccionado sus elementos y los ha jerarquizado de acuerdo a sus necesidades, en Guatemala existen cuatro etnias y 25 grupos culturales (22 mayas, un garífuna, un xinca y mestizo) con diferentes idiomas, costumbres y creencias mágico-religiosas. Entre los mayas, la práctica médica o el arte de curar alcanzó altos niveles de desarrollo, seguramente por las grandes epidemias de que fueron víctimas, por lo que se sintieron obligados a encontrar los recursos necesarios para aliviar sus dolencias. Como en otras culturas antiguas, la civilización maya muestra el carácter sagrado de la medicina. Los mayas desarrollaron amplios conocimientos sobre la flora y la fauna de las tierras que habitaron, lograron seleccionar y aprovechar todas aquellas a las que les descubrieron propiedades terapéuticas. Muchas de las plantas utilizadas con fines terapéuticos en la cultura maya, son actualmente motivo de estudio, con la finalidad de conocer su composición química y sus propiedades farmacológicas (Cleaves, 1999; Eder \& García, 2003; Taracena, 1983; Thompson 1977).

El conocimiento de las muchas enfermedades que afectaban a la población nativa y su cura a través de uso de plantas, animales y minerales fue una de las grandes virtudes de los antiguos pueblos de Guatemala, conocimiento que a pesar de varios intentos por erradicarlo o desaparecerlo ha perdurado hasta nuestros días (Villatoro, 1984).

La conquista llevada a cabo por los españoles en Guatemala fue una época de cambios bruscos, en donde los indígenas tuvieron que transformar sus costumbres, ritos, religión y autoridades. Varios autores indican que poco después de la conquista hubo en toda Mesoamérica una lamentable y brusca pérdida de los conocimientos. La destrucción de códices y la muerte de los científicos y sabios locales por enfermedades y violencia fueron pérdidas irreparables. Pero la civilización de los pueblos indígenas mantuvo y ha mantenido sus conocimientos ancestrales en el uso de plantas medicinales, por lo que en la constante lucha de dominación hubo una fusión de conocimiento, en donde ganaron ambos grupos. En el período colonial la medicina tradicional conservó rasgos muy similares, a los de la época prehispánica, tanto en la concepción de las enfermedades como en las técnicas y recursos empleados para el tratamiento de las mismas (Eder \& García, 2003; Figueroa, 1955; Gómez-Pompa, 1993; Morley, 1980; Orellana, 1987). 
Para enfrentar las brechas existentes derivadas de condiciones históricas, económicas, sociales y políticas, es necesario iniciar un proceso de cambio para contribuir a mejorar la calidad de vida de la población, establecer estrategias funcionales que permitan obtener mayores beneficios, reducir riesgos y optimizar resultados. Hay que tomar en consideración la equidad en la distribución de recursos dirigidos a la atención primaria de la salud, ofreciéndole a la población guatemalteca el acceso universal a los servicios con un enfoque eficaz, incluyente y digno.

Tomando en cuenta el perfil epidemiológico que se presenta en el país, la educación y la promoción de la salud se convierten en una estrategia importante para impulsar la cultura de la prevención y mantenimiento de la salud. El análisis presupuestario del MSPAS señala la necesidad de asignar un presupuesto específico para dichas actividades, y la necesidad de mejorar la coordinación intersectorial para hacer más eficiente el gasto público.

La interculturalidad es una oportunidad para que el sistema médico oficial también aprenda del sistema médico indígena y que este último pueda aportarle al primero conceptos y herramientas que permitan mejorarlo. Hay que asumir que el sistema médico oficial no es el único llamado a "salvar" a los pueblos indígenas, incorporándolos a sus propios referentes, a sus ideas de modernidad, de conocimiento científico, de universalismo; sino que es posible que también se aprenda del sistema indígena (Cotton, 2004).

Existe un enfoque de derechos en la salud que es concomitante al reconocimiento por la mayoría de los países de la diversidad cultural de los pueblos indígenas y a los derechos específicos en materia de salud indígena, acorde a dos instrumentos fundamentales, los cuales se encuentran en los Arts. 6, 24 y 25 del Convenio 169 de la Organización Internacional del Trabajo (OIT) (obligación del Estado a extender progresivamente la seguridad social a los pueblos indígenas y la responsabilidad de que los servicios de salud sean adecuados y tomen en cuenta las medicinas tradicionales), y la iniciativa Sapia de la Organización Panamericana de la Salud OPS/OMS, que establece un conjunto de principios para el trabajo con las comunidades indígenas: El abordaje integral de la salud, la autodeterminación de los pueblos indígenas, la participación sistemática, el respeto y la revitalización de las culturas indígenas y la reciprocidad en las relaciones (Cotton, 2004).

Estos derechos avalan la necesidad de un desarrollo institucional apropiado y que los servicios de salud se centren en las personas a quienes se les deben reconocer sus derechos específicos, tanto como personas individuales como a colectividades específicas que poseen una identidad particular. En este contexto, el bienestar es concebido por los indígenas como: la armonía de todos los elementos que hacen la salud, es decir el derecho a tener su propio entendimiento y control de su vida, y el derecho a la convivencia armónica del ser humano con la naturaleza, consigo mismo y con los demás, encaminada al bienestar integral, a la plenitud y tranquilidad espiritual, individual y social (Cotton, 2004; Villatoro, 1984).

En ese sentido, el enfoque intercultural de la salud, es una de las estrategias para mejorar el acceso y la calidad de la atención y la promoción de la salud, es también una de las estrategias para proporcionar a los pueblos los medios necesarios para mejorar su salud y tener mayor control de sus vidas. El tema de la interculturalidad ha traspasado los límites de la educación y empieza a asentarse en el ámbito de la salud. La discusión y acciones en torno a la problemática de salud y pueblos indígenas, ha generado, al menos cuatro enfoques (Cotton, 2004):

El enfoque que surge al interior de los servicios oficiales de salud, se asume liberal y declara que los usuarios son iguales, sean éstos indígenas o no indígenas, bajo esa premisa rechazan cualquier posibilidad de considerar las especificidades culturales y sociales de la población indígena, a la hora de definir e implementar programas de salud.

El enfoque que señala que hay "variables culturales" en los indígenas que condicionan la situación crítica de la salud. Dichas variables son vistas más como barreras a superar que como una potencialidad a desarrollar. En ese sentido, se tiende a una instrumentalización de elementos culturales; de forma que, el problema se centra a nivel de la comunicación. Se trata de mejorar tales relaciones, exigiendo a los pacientes indígenas una adherencia absoluta y total a los programas que se implementan.

El enfoque de algunas organizaciones indígenas, que plantean el rescate y la revalorización de la medicina tradicional, se centra en el sistema de salud como elemento de identidad étnica; sin embargo, olvida que los indígenas de hoy no son los mismos de antes, que no existe una única identidad indígena, existe un pueblo disperso que se posesiona en distintos pisos ecológicos $\mathrm{y}$, según donde se posesione, se identifica como indígena o como campesino.

El enfoque que busca articulaciones entre el sistema médico indígena y el sistema médico oficial. Busca desarrollar espacios interculturales para el desarrollo regional con un enfoque sectorial. Asume que existe una 
interrelación práctica entre los problemas de salud y los distintos elementos del desarrollo social (Cotton, 2004).

Una experiencia que se tiene en Guatemala es el trabajo desarrollado por Médicos Descalzos de Guatemala, la cual está avocada al desarrollo endógeno de la Medicina Tradicional en las áreas rurales. Se propone rescatar los conocimientos tradicionales en salud herbolaria, mejorar la accesibilidad del primer nivel de atención con la incorporación de la medicina natural herbolaria de acceso local. También pretende aumentar la participación comunitaria en la resolución de los problemas básicos de salud y promueven la articulación entre los actores de la medicina tradicional y el sistema integral de atención en salud.

Otra experiencia consolidada es la de la Asociación de Servicios Comunitarios de Salud (Asecsa), que difunde servicios interculturales en varios municipios indígenas, siendo una de sus actividades más sobresalientes la capacitación. Actúan básicamente a través de organizaciones de base como Cosprek, Aprosade y Aprosadec. También son destacables los proyectos de la Asociación CDRO para el fortalecimiento de la medicina indígena y sobre todo la diseminación de medicamentos indígenas mediante la red de farmacias de CDRO (Eder \& García, 2003).

A principios del 2012 se definió en el Sistema Integral de Atención en Salud (Sias), material que impulsa la estrategia de municipios saludables, definida como aquel municipio donde todas sus autoridades, instituciones, organizaciones y ciudadanos trabajan conjuntamente para mejorar la salud y la calidad de vida de su población, mediante la promoción y el fortalecimiento de la participación ciudadana; la promoción e implementación de políticas públicas saludables; el mejoramiento de los ambientes y entornos; la promoción de prácticas de vida saludables y la mejora continua de la calidad de sus servicios públicos (salud, educación, etcétera.). Sin embargo, la estrategia municipios saludables, aunque es una estrategia de promoción encaminada a desarrollar aquellos elementos de los determinantes de la salud que son responsabilidad de los gobiernos y sociedad civil, no tiene respaldo presupuestario.

Es necesario priorizar el gasto, enfocar la investigación en las patologías más frecuentes por región, aprovechar esa biodiversidad con que cuenta el país para el desarrollo de productos naturales medicinales, orientarse a la promoción de la salud y prevención de enfermedades como lo indica el diagnóstico realizado por el MSPAS en el 2012. Es recomendable una política de desarrollo del recurso humano que permita la dig- nificación del personal de la salud y reducir la tasa de rotación del personal, así como fortalecer los servicios del sector salud con perspectiva de género y pertinencia cultural. Se recomienda introducir dentro del sistema de salud un programa de mejoramiento continuo de la calidad, que permita mejorar las condiciones en que se brinda y se recibe la atención de salud, mejorar sustancialmente el financiamiento de la salud por parte del Estado para disminuir el gasto.

Se plantea la necesidad de redefinir y reasignar de manera prioritaria las metas contenidas en los Acuerdos de Paz, con la finalidad de superar en el mediano plazo las brechas que se generan en el financiamiento de la salud. El tema del financiamiento de la salud en Guatemala debe institucionalizarse y monitorearse a través de las cuentas nacionales de salud, de tal forma que la información dé respuestas a las instancias políticas de coyuntura. Se resalta el implementar y garantizar el funcionamiento y sostenibilidad del Modelo Básico de Salud (MBS) para fortalecimiento del primer nivel de atención y la estrategia de Atención Primaria de Salud Renovada de la OMS, así como institucionalizar las perspectivas de género y étnicas en materia de salud e implementar la estrategia mundial de medicina tradicional de la OMS.

Es importante señalar aspectos prioritarios que se deben modificar dentro del sistema (en el nivel local) para mejorar su calidad y eficiencia. Como lo es la participación social para que en conjunto se asuma la salud como una opción de todos y un objetivo dentro de una perspectiva de desarrollo en el contexto histórico y sociocultural local, donde se conozca la realidad de salud local, se definan los intereses y soluciones a las dificultades presentes de forma coordinada y planificada.

Se debe de planificar en la medida de lo posible de manera conjunta con los sectores involucrados, en cada uno de los subcomponentes del sistema de salud y socializar los planes para incorporarlos al desarrollo local, desde los conceptos y percepciones de salud y enfermedad para que respondan a la problemática local.

En los sistemas de salud de todo el mundo, los niveles de enfermedades crónicas y los costos de atención sanitaria son cada vez más elevados. Tanto los pacientes como los dispensadores de atención de salud están exigiendo la revitalización de los servicios de salud y haciendo hincapié en la atención individualizada centrada en la persona. La recopilación, el análisis y la síntesis de datos sobre la utilización de la MTC son prioridades esenciales, junto con una estrategia de gestión de la información que asegure su actualización y 
su máxima calidad. Esto se puede conseguir mediante la inclusión, en las encuestas nacionales, de preguntas sobre la incorporación de la MTC; la utilización de bases de datos existentes; el establecimiento de centros de investigación especializados; o el desarrollo de redes nacionales de investigación y de múltiples partes interesadas (OMS, 2013).

\section{Conclusiones}

La medicina tradicional y la fitoterapia deben constituirse como una alternativa que se debe integrar al sistema de salud para lograr mayor accesibilidad y cobertura principalmente en la atención primaria.

El uso de las plantas medicinales y productos naturales medicinales en el contexto de la fitoterapia clínica permite abordar el tratamiento de las afecciones más comunes, por lo que constituye una medicina complementaria de acción y de asociación terapéutica posible.

El uso apropiado de los productos naturales sobre bases científicas, teniendo en cuenta los conocimientos actuales y la integración de la medicina tradicional y fitoterapia clínica en el sistema de salud, puede ayudar a resolver problemas relacionados con el costo de la salud y responder a la demanda de la población.

Preconizar o promover el uso de la medicina tradicional y productos naturales sin integrar este saber dentro de los conocimientos científicos y médicos actuales, y si las prácticas no se realizan dentro de un abordaje clínico integrativo, sólo puede revelar rápidamente sus deficiencias y terminar en el abandono o desprestigio dichas terapias y productos.

El avance científico de la medicina tradicional y fitoterapia estimula su desarrollo y propagación en la población, dándole un realce a las opciones terapéuticas disponibles para distintas enfermedades pero basados en la evidencia.

Es importante resaltar que la utilización de los productos naturales medicinales, consideran diversas terapias como la fitoterapia, zooterapia, micoterapia y mineraloterapia, que es la forma en que se está regulado su uso actualmente en Guatemala y está en proceso de armonización a nivel centroamericano.

Resulta de importancia incluir en los sistemas de salud tanto las prácticas, como a los profesionales capacitados para ejercer este tipo de terapias, que a largo plazo, permitan alcanzar un estándar de calidad para que sea una alternativa viable para el mejoramiento de la salud en Guatemala y no sea concebida como algo que practican solamente los indígenas, sino que esté incorporada dentro del sistema nacional de salud al alcance de toda la población.

\section{Referencias}

Balick, M. J. (1990). Ethnobotany and the identification of therapeutic agents from the rainforest. En D. J. Chadwic \& J. Marsh (Eds.), Bioactive compounds from plants (pp. 22-31). Ciba Foundation Symposium 154, Chichester: John Wiley \& Sons.

Bonati, A. (1990). How and why should we standardize phytopharmaceutical drugs for clinical validation?. Journal of Ethnopharmacology, 32(1-3), 195-197. doi 10.1016/0378-8741(91)90117-V

Bruhn, J. \& Holmstedt, B. (1981). Ethnopharmacology: Objectives, principles and perspectives. En J. L. Beal \& E. Reinhard (Eds.), Natural products as medicinal Plants (pp 405-430). HippocratesVerlarg, Stuttgart, Germany.

Cáceres, A. (1996). Plantas de uso medicinal en Guatemala. Guatemala: Editorial Universitaria.

Cáceres, A. (2009). Vademécum Nacional de Plantas Medicinales. Guatemala: Editorial Universitaria. 313 p.

Cañigueral, S. (2002). La Fitoterapia: ¿Una terapéutica para el tercer milenio? Revista de Fitoterapia, 2(2), 101-121.

Cañigueral, S. \& Vila, R. (1998). Fitoterapia: Concepto, límites y fuentes de información. En A. Arteche, B. Vanaclocha, J. I. Güenechea, \& R. Martínez (Eds.). Fitoterapia. Vademécum de prescripción: Plantas medicinales ( $3^{\mathrm{a}}$ ed.). Barcelona: Masson.

Cañigueral, S. \& Vila, R. (2003). La Fitoterapia racional. En B. Vanaclocha \& S. Cañigueral (Eds.). Fitoterapia. Vademécum de prescripción ( $4^{\mathrm{a}} \mathrm{ed}$.). Barcelona: Masson.

Cleaves, C. (1999). Medicina que está en las plantas. Salud preventiva y uso de plantas medicinales en el alivio o cura de algunas de las enfermedades más comunes en Guatemala. Guatemala: Ediciones CEIBAS.

Cotton, H. L. (2004). Análisis crítico del Sistema Nacional de Salud en Guatemala (Tesis de maestría). Universidad Rafael Landívar, Quetzaltenango, Guatemala.

Eder, K. \& García, M. M. (Coord.). (2003). Modelo de la medicina indigena maya guatemalteca: Investigación participativa en Sipacapa, San Marcos, San Martín Jilotepeque, Chimaltenango y San Juan Ixcoy Huehuetenango ( $2^{\mathrm{a}}$. ed.). Guatemala: Asociación de Servicios Comunitarios de Salud. 
Figueroa, H. (1955). Enfermedades de los conquistadores. San Salvador, El Salvador: Ministerio de Cultura, Departamento Editorial.

Gómez-Pompa, A. (1993). Las raíces de la etnobotánica mexicana. En S. Guevara, P. Moreno-Casasola, \& J. Rzedowski (Comp.), Logros y perspectivas del conocimiento de los recursos vegetales de México en vísperas del Siglo XXI (pp. 26-37). Xalapa, Veracruz, México: Instituto de Ecología y Sociedad Botánica de México.

Martínez, C. (1964). Las ciencias médicas en Guatemala, origen y evolución ( $3^{\mathrm{a}}$. ed.). Guatemala: Editorial Universitaria.

Mendoza, N., de León, J., \& Figueroa-Hernández, J. L. (2005). Herbolaria. Revista Facultad de Medicina UNAM, 48(6), 248-249

Ministerio de Salud Pública y Asistencia Social. (2012). Diagnóstico Nacional de Salud. Guatemala: Autor. Recuperado de http://www.mspas.gob. gt/files/Descargas/UNIDADES/CuentasNacionalesSalud/Publicaciones/11\%20MSPAS $\% 20$ $\% 282012 \% 29 \% 20$ Diagnostico-Salud-marzo.pdf

MSPAS. (2011). Encuesta Nacional de Salud Materno Infantil 2008 (ENSMI-2008/09). Ministerio de Salud Pública y Asistencia Social (MSPAS)/Instituto Nacional de Estadística (INE)/Centros de Control y Prevención de Enfermedades (CDC). Guatemala.

Morley, S. G. (1980). La civilización Maya. México: Fondo de Cultura Económica.

Muñoz, G. (19 de febrero de 2016). Salud confirma 90 casos de Zika. Prensa Libre. Guatemala.

Obregón, L. (2000). Fitoterapia: Una alternativa al servicio de los pueblos en el tercer milenio. En $\mathrm{Me}$ moria del I Curso Iberoamericano de Fitoterapia Clínica y III Reunión de Coordinación Internacional de RIPROFITO (Red Iberoamericana de Productos Fitofarmacéuticos). Guatemala: Cyted, Conaplamed y Concyt.
Organización Mundial de la Salud. (1991). Programa de medicina tradicional. Ginebra, Suiza: Autor

Organización Mundial de la Salud. (2002). Estrategia de la OMS sobre medicina tradicional 2002-2005. Ginebra, Suiza: Autor.

Organización Mundial de la Salud. (2013). Estrategia de la OMS sobre medicina tradional 2014-2023. Ginebra. Suiza: Autor.

Orellana, S. (1987). Indian medicine in highland Guatemala: The pre-Hispanic and colonial periods. Albuquerque: University of New Mexico Press.

Secretaría de Planificación y Programación de la Presidencia, \& Ministerio de Salud Pública y Asistencia Social (2011). Estudio Nacional de Mortalidad Materna 2007. Guatemala: Serviprensa.

Taracena, J. A. (1983). La expedición cientifica al reino de Guatemala. Guatemala: Editorial Universitaria.

Thompson, J. E. (1977). Historia y religión de los Mayas (2 $2^{\mathrm{a}}$. ed.). México: Siglo Veintiuno.

Tyler, V. E. (1999). Phytomedicines: Back to the future. Journal of Natural Products, 62(11), 1589-1592. doi: 10.1021/np9904049

Villatoro, E. M. (1982). Vida y obra de los curanderos de Petén. La Tradición Popular, 38, 1-18.

Villatoro, E. M. (Comp.). (1984). Etnomedicina en Guatemala. Guatemala: Centro de Estudios Folklóricos. 